\title{
RFRSN: Improving protein fold recognition by siamese network
}

\author{
Ke Han, Yan Liu, and Dong-Jun Yu*
}

Keywords: protein fold recognition; bioinformatics; convolutional neural network

\section{Author Biography:}

Ke Han received her M.S. degree in computer science from Nanjing University of Science and Technology in 2009. She is currently a PhD candidate in the School of Computer Science and Engineering at Nanjing University of Science and Technology and a member of Pattern Recognition and Bioinformatics Group. Her research interests include pattern recognition, machine learning and bioinformatics.

Yan Liu received his M.S. degree in computer science from Yangzhou University in 2019. He is currently a $\mathrm{PhD}$ candidate in the School of Computer Science and Engineering at Nanjing University of Science and Technology and a member of Pattern Recognition and Bioinformatics Group. His research interests include pattern recognition, machine learning and bioinformatics.

Dong-Jun Yu received the PhD degree from Nanjing University of Science and Technology in 2003. He is currently a full professor in the School of Computer Science and Engineering, Nanjing University of Science and Technology. His research interests include pattern recognition, machine learning and bioinformatics. He is a senior member of the China Computer Federation (CCF) and a senior member of the China Association of Artificial Intelligence (CAAI). 


\section{ABSTRACT}

Protein fold recognition is the key to study protein structure and function. As a representative pattern recognition task, there are two main categories of approaches to improve the protein fold recognition performance: 1) extracting more discriminative descriptors, and 2) designing more effective distance metrics. The existing protein fold recognition approaches focus on the first category to finding a robust and discriminative descriptor to represent each protein sequence as a compact feature vector, where different protein sequence is expected to be separated as much as possible in the fold space. These methods have brought huge improvements to the task of protein fold recognition. However, so far, little attention has been paid to the second category. In this paper, we focus not only on the first category, but also on the second point that how to measure the similarity between two proteins more effectively. First, we employ deep convolutional neural network techniques to extract the discriminative fold-specific features from the potential protein residue-residue relationship, we name it SSAfold. On the other hand, due to different feature representation usually subject to varying distributions, the measurement of similarity needs to vary according to different feature distributions. Before, almost all protein fold recognition methods perform the same metrics strategy on all the protein feature ignoring the differences in feature distribution. This paper presents a new protein fold recognition by employing siamese network, we named it PFRSN. The objective of PFRSN is to learns a set of hierarchical nonlinear transformations to project protein pairs into the same fold feature subspace to ensure the distance between positive protein pairs is reduced and that of negative protein pairs is enlarged as much as possible. The experimental results show that the results of SSAfold and PFRSN are highly competitive. 


\section{INTRODUCTION}

As the genome project continues to evolve, we are faced with exponentially growing sequences of proteins without knowing their structural or biochemical functions. Exploring the structure and function of even a single protein remains a non-trivial task, the best way to understand all these sequences is to search a database and link them to other proteins with known correct structures, which is also the goal of protein fold recognition. Improving these methods of protein fold recognition is one of the fundamental challenges in bioinformatics today. In general, these methods of protein fold recognition can be divided into machine learning methods and alignment methods.

Machine learning methods first extract fold-specific features and then directly classify proteins into different fold categories by employing different classifiers. In the early work, support vector machine and neural network [1] have been employed to construct a single classifier to identify fold type. Shen et al. [2] used ensemble classifiers to improve protein fold pattern recognition. Liu et al. [3] proposed SOFM to extract the sequence-order information of neighboring residues from multiple sequence alignment (MSA). Later, the RF-fold [4] and DN-fold [5] have been proposed by combining the deep neural network (DNN), random forest (RF) [6] and various features describing the pairwise similarities of two different protein sequence.

In contrast to machine learning methods, the mechanism of the alignment methods is that fold types are identified based on the similarity between the query protein and template at sequence-sequence [7-10] or sequence-structure level $[11,12]$. The sequence of a query protein is aligned against the sequences of template proteins whose folds are known to generate similarity scores. If the similarity scores between a query protein and a template protein is the highest one of all similarity scores, and then the fold type of the template protein is considered as the fold type of the query protein.

All of the methods mentioned above are driving the development of this important field, they focus on employing discriminative frameworks to extract a robust and discriminative protein descriptor, which is used to measure the similarity by hand-crafted distance metrics, such as Euclidean distance and Cosine distance. But there are also suffering from the following shortcomings: Similarity measures of protein feature are not rigorous because different protein 
feature usually subject to varying distributions, if we perform the same metrics on all the feature, the differences in feature distribution will be ignored. In addition, in the case of higher feature dimension, the distance between samples tends to be the same, so it is hard to measure the distance between different samples. To address these problems, based on the idea of metrics learning, we propose a new protein fold recognition by employing siamese network, we named it PFRSN. The objective of PFRSN is to learn a set of hierarchical nonlinear transformations to project protein pairs into the same fold feature subspace to ensure the distance between positive protein pairs is reduced and that of negative protein pairs is enlarged as much as possible. In addition, RFRSN is also dependent of the protein feature representation, robust and comprehensive feature representations contribute to the performance of RFRSN.

Recently, Zhu et al. [13] proposed a new protein descriptor called DeepFR to extract the fold-specific features by using deep convolutional neural network (DCNN) from protein residue-residue contact map and it improve the accuracy of protein fold recognition. However, DeepFR suffers from the following shortcomings: (1) what we found in our experiments shows that the potential relationship between protein residues is lost by pass the contact likelihood matrix extracted by CCMpred [14] through DCNN, because the contact likelihood matrix were filtered by activation function. (2) Multiple sequence alignment is required when using CCMpred to predict protein residue contact map, it is time-consuming and very inconvenient for performing protein fold recognition. In order to overcome these shortcomings, we use SSA tool [15] (A fast protein residue contact map prediction tool that requires only sequence as input) instead of CCMpred to predict the potential relationship between protein residues (Output of the previous layer of the SSA model), this potential relationship is native and not filtered by the activated function, which contains both protein residue-residue contact information and other protein structure information. On the other hand, we design a new network structure to make it effectively mine the structure information hidden in the potential relationship between protein residues. To distinguish it with DeepFR, we name it SSAfold.

In summary, the main contributions of our study are as follows: 
(1) The idea of metrics learning was introduced into protein fold recognition to fill the gap in this point;

(2) Siamese networks are used to learn the complex nonlinear relationships stored in protein feature so that they can better measure the similarity between any two proteins in the protein fold subspace;

(3) The ability of DeepFR to extract protein feature was accelerated and improved by using the potential relation between protein residues alternative the protein contact map as the input of convolutional neural network and improving the structure of neural network.

The rest of this paper is organized as follows. We give a brief background on metrics and deep learning in section 2. The effectiveness analysis and the proposed SSAfold and RFRSN are presented in section 3. Experiment results are provided in section 4. Finally, we give a conclusion in section 5 .

\section{MATERIALS AND METHODS}

\section{Benchmark datasets}

121 In this paper, we train our SSAfold model and RFRSN by employing the SCOP2.06 dataset [16, 17]. In addition, to ensure the independence of training data and test data, the training set should be cleaned to remove the proteins that have significant sequence similarity with proteins in test dataset. CD-HIT_2D [18] is employed to guarantee all the proteins in the database share $40 \%$ sequence similarity with the proteins in test dataset. After removing the sequence redundancy in the training set, finally, we collected a training dataset consists of 23001 proteins covering 1198 folds, 1948 superfamilies and 4646 families.

\section{Test dataset}

130 (version 1.37) with pairwise sequence identity less than 40\%. In LINDAHL dataset, 321, 434 and

131555 proteins have at least one match at fold, superfamily and family levels, respectively.

\section{Metrics learning}


134 similarity among samples pairs while using an optimal distance metric for learning tasks. Original metric learning approcaches learns a linear Mahalanobis distance metric for similarity measurement [20-22]. For example, Weinberger et al. [23] proposed a large margin nearest neighbor method named LMNN by enforcing an anchor sample to share the same labels with its neighbors by a relative distance, which is one of the most popular metric learning methods before. Davis et al.[24] presented an metric learning (ITML) method based on information theoretic, which contributes multivariate Gaussian distribution and Mahalanobis distances into an information-theoretic setting. However, these methods only learn an ensemble of linear projections and cannot fully learn the nonlinear relationships hidden in the data, which are quite common in the real world applications. To address this problem, many methods based on kernel tricks [25-27] are usually employed for nonlinear transformations, yet they cannot determine the specific function and face scalability problem for other tasks. Recently, with the development of deep learning and several deep metric learning methods have been presented to address the limitation of kernel method by learning hierarchical nonlinear transformations [28, 29]. For example, $\mathrm{Hu}$ et al. [28] proposed a discriminative metric learning method (DDML) to learn the distance between faces. denoising method by using deep convolutional neural networks with batch renormalization. Mun et al.[34] considered the temporal dependency of the events into the deep convolutional neural networks for dense video caption. In addition, deep learning has achieved impressive success in various tasks in the field of bioinformatic. For example, Li et al. [35] proposed ResPRE model, which is a high-accuracy protein contact prediction tool by coupling precision matrix with deep residual neural networks; Differently, our proposed RFRSN method employs a siamese network to learn the nonlinear distance metric and we use the back propagation algorithm to train the model.

\section{The proposed RFRSN model for fold recognition}


In this section, we propose a new method (RFRSN) for protein fold recognition, where the basic idea of RFRSN is illustrated in Fig. 1. We use a siamese network to map a pair of proteins feature into the same fold subspace, where the semantic distance of protein features can be directly simulated by the Euclidean distance in this subspace. The choice of feature descriptors is unlimited, the existing powerful protein feature descriptors can be used directly. For get better performance, we also propose a robust and discriminate protein feature descriptor named SSAfold in this paper, which can extract feature from potential protein residue relationship by using deep convolutional neural network. Next, we present the proposed SSAfold method and RFRSN model, as well as its implementation details.

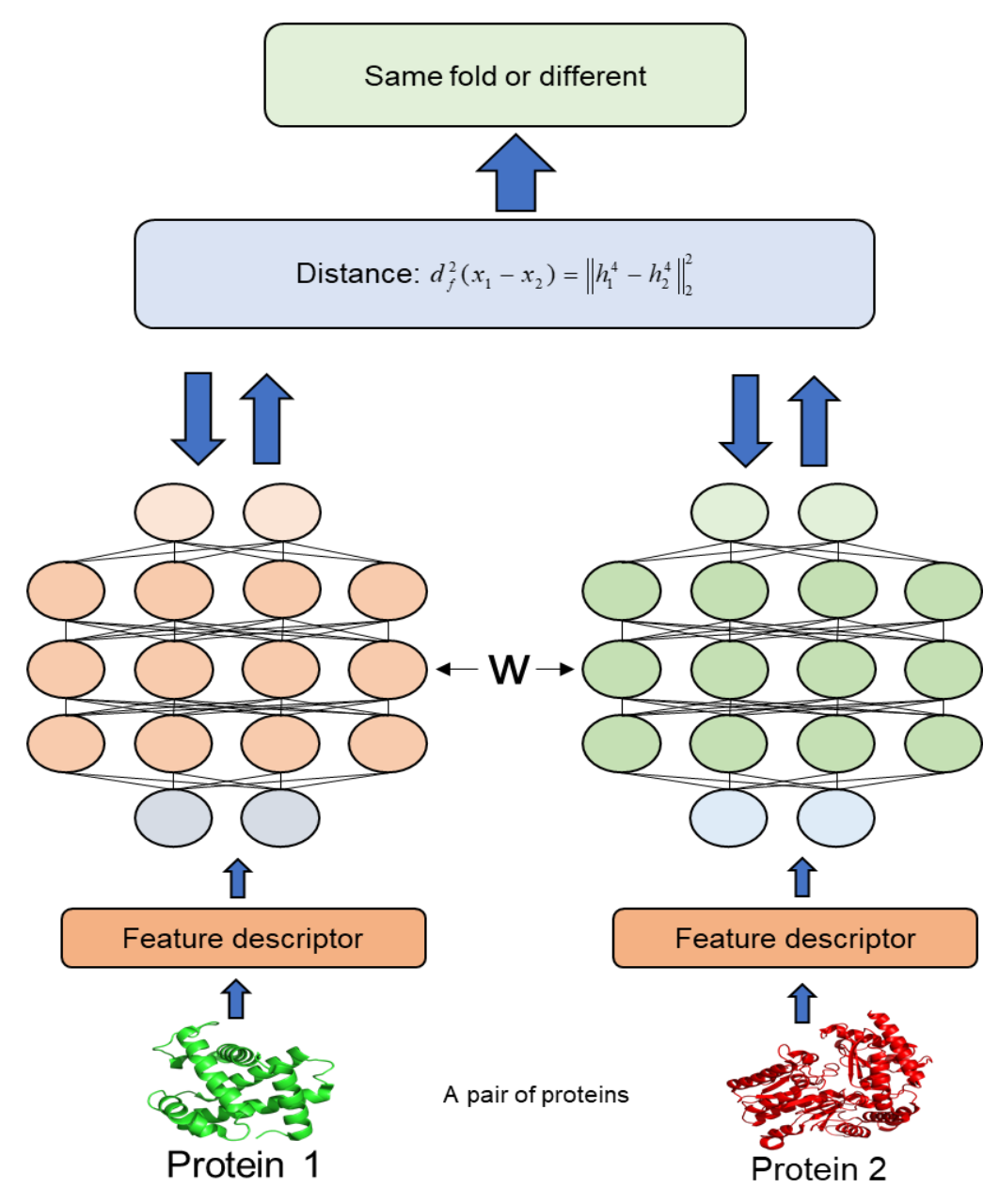

172 Fig.1. The flowchart of proposed RFRSN method for protein fold recognition. For a given pair of 173 feature vector of protein 1 and protein 2, they are mapped into the same fold subspace as $H_{1}^{4}$ and 
$H_{1}^{4}$ and $H_{2}^{4}$ is calculated and employed to determine whether two proteins come from the same

SSAfold: a fast and discriminate protein feature descriptor from predicting potential protein residue-residue relationship

There are many methods to predict protein residue-residue contacts, for example DeepCov [36], DNCON2 [37], DeepCON [38] and ResPRE [35]. These methods can produce very accurate residue-residue contacts, however, for these methods, homologs to the query protein must be collected by running HHblits [39] to search against sequence database UniProt dataset and then were organized as an MSA of the query protein. However, it takes a lot of time. Due to the limitation of our computer, we choose the SSA method as the potential protein residue-residue relationship extractor of SSAfold, SSA is very fast and accurate, which requires no information other than protein sequence (details about SSA can be seen in paper [15] ). Originally, SSA maps any protein sequence to a sequence of vector embeddings - one per amino acid position - that encode structural information and outputs residue-residue contacts. In this paper, the parameters of SSA provided by the author of SSA and we only employ the previous layer output of residue-residue contact as the potential protein residue-residue relationship.

\section{Extracting fold-specific features from potential protein residue-residue relationship}

The acquired residue-residue relationship is difficult to directly infer fold type of query protein, the main reasons are as follows: (1) although residue-residue relationship matrices contain a lot of structural information, it also contains a large amount noise and redundant information. (2) Due to the length of the protein sequence may not be the same, the similarity scores between two protein sequences are difficult to obtain. To sum up, how to use potential protein residue-residue relationship effectively for protein fold recognition is still a huge challenge.

Inspired by the tremendous success of convolutional neural networks in computer vision, we employ deep convolutional neural networks to extract fold-special feature from potential protein residue-residue relationship. Specifically, the DCNN takes predicted potential protein residue-residue relationship matrix of a query protein as input, and outputs fold type of the query 
protein residue-residue relationship matrices of a protein together with its fold type as the label. The whole training process minimizes the cross entropy loss function through backpropagation [40].

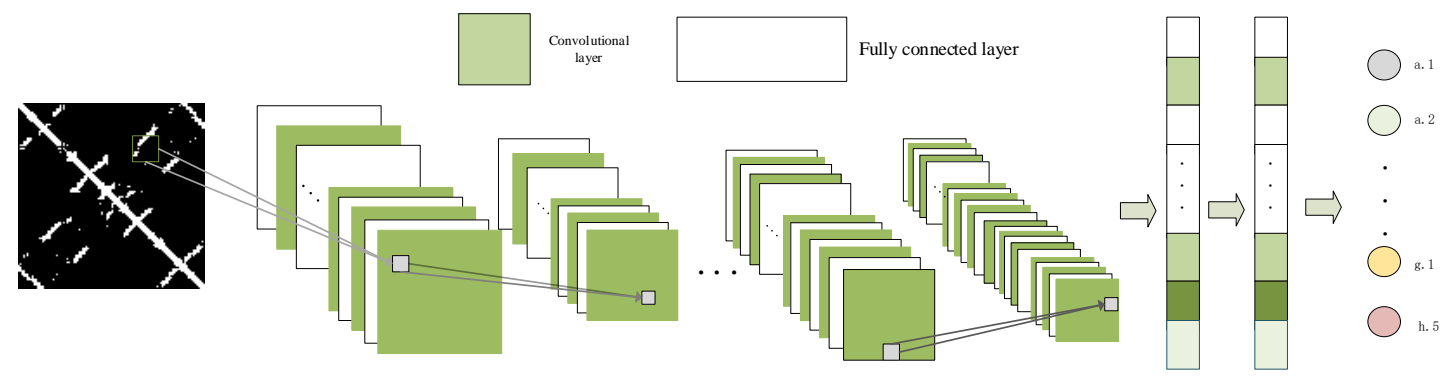

Fig. 2. Architecture of the deep convolutional neural network used to extract fold-specific features from potential protein residue-residue relationships.

Architecture of the deep convolutional neural network is shown in Fig. 2, which includes thirteen convolutional layers, three max-pooling layers, thirteen batchnorm layers and three fully connected layers. The parameters of SSAfold are given in Supplementary in formation S1.

For the full connected layers of SSAfold, the size of the input data must be the same, however different protein sequences usually have different sequence lengths $L$. According to the output of the SSA model, the size of potential protein residue-residue relationship matrice is $L \times L$. In order to solve this contradiction, we fix the size of the residue-residue relationship matrice is $256 \times 256$ by adopting sampling or padding operations accordingly, these two operations are widely used in the field of computer vision [41]. The specific sampling and padding strategies are described as follow:

- Sampling: For the length of protein over 256 , we randomly sampled a $256 \times 256$ sub-matrix from its potential protein residue-residue relationship matrix. We repeated this operation ten times and obtained an ensemble.

- Padding: For the length of protein shorter than 256, we embedded its relationship matrix into a $256 \times 256$ matrix with all elements being 0 . The embedding positions are random; thus, we obtained an ensemble of $256 \times 256$ matrices after repeating this procedure ten times.

Extracting fold feature by SSAfold: to our best knowledge, the fully connected layers play the role of "classifier" in the whole convolutional neural network. If operations such as convolution layer, pooling layer and activation function layer map the original data to the hidden feature space, the full connected layer maps the learned "distributed feature representation" to the sample space. In 
this paper, we use the output of the first fully connected layer were used as the input of metric Learning Network, we named it SSAfold features. SSAfold feature has the comprehensive information and they are higher-level features made up of lower-level features. Experiments show that this strategy can get the best results.

We use the SSAfold features mentioned in the previous section as the protein feature descriptor.

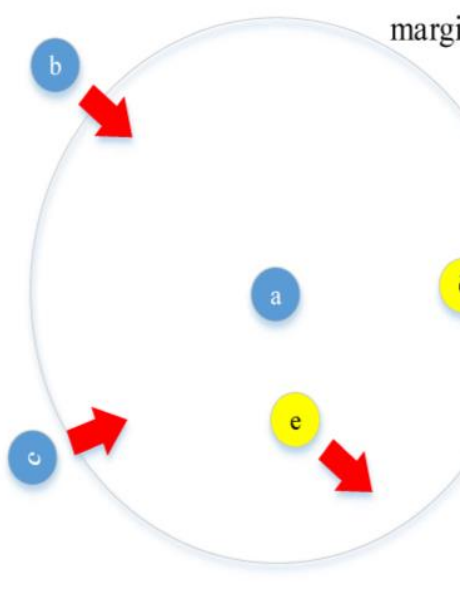

before

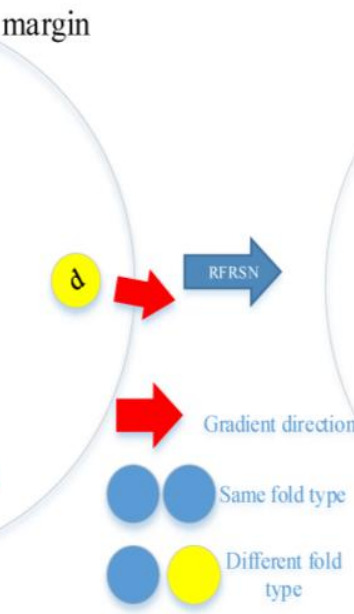

type

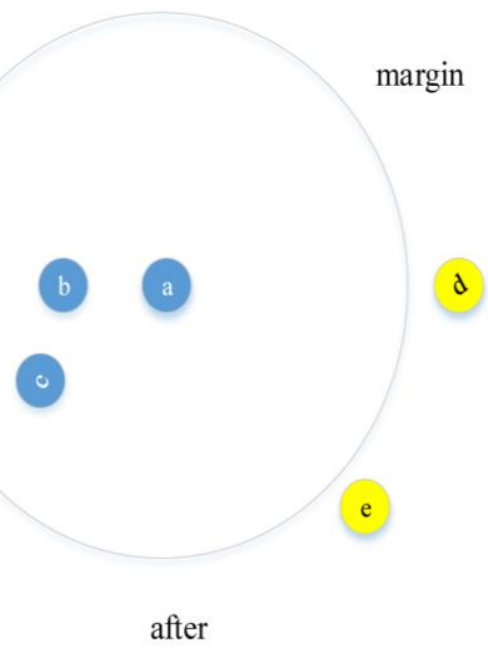

after

Fig. 3. Intuitive illustration of the proposed RFRSN method. There are five protein sequence, which $\mathrm{a}, \mathrm{b}$ and $\mathrm{c}$ belong to the same protein fold type, and $\mathrm{d}$ and e belong to the same protein fold type, here, assume protein a as anchor protein. In the original protein feature space, the distance between the positive pair is larger than that between the negative pair which may be caused by the individual differences of different protein. This phenomenon is not conducive to protein fold recognition. Then, we use our proposed RFRSN to create a gradient that pulls positive protein closer to the anchor protein and push the negative protein away from the anchor protein. FInally, the distance of each positive protein pair is less than the margin and that of each negative protein pair is higher than the margin. 
parameters) to compute the feature representation of a protein pair by passing them through multiple layers of nonlinear transformations. Now, assume the number layers of deep neural network is set to $\mathrm{M}+1$, and each layer has $p^{m}$ hidden points, where $\mathrm{m}=1,2, \ldots, \mathrm{M}$. For a given protein $x \in R^{d}, \mathrm{~d}$ is the dimension of original protein feature. The output of first layer is $h^{1}=s\left(w^{1}+b^{1}\right)$, where $w^{1}$ and $b^{1}$ is the parameters of the first layer to be learned in training process and $\mathrm{s}$ is the nonlinear activation function, such as relu and sigmoid. Then, the first output is used to be as the input of next layer, we repeat this operation and get the output of the m-th layer: $h^{m}=s\left(w^{m} h^{m-1}+b^{m}\right)$, where $w^{m}$ and $b^{m}$ is the parameters of the m-th layer. Finally, the output of the top level can be computed as: matrix $w^{m}$ and bias $b^{m}$, where $\mathrm{m}=1,2, \ldots, \mathrm{M}$.

Now, given a pair of protein sample $x^{i}$ and $x^{j}$, pass them into the siamese network respectively. Finally, they can be represented as $f\left(x^{i}\right)=h_{i}^{M}$ and $f\left(x^{j}\right)=h_{j}^{M}$. The distance of protein pair can be measured by computing the squared Euclidean distance between the most top level representations, which can be defined as follows:

$$
f(x)=h^{M}=s\left(w^{M} h^{M-1}+b^{M}\right)
$$

Where the mapping project $f: \mathrm{R}^{d} \rightarrow \mathrm{R}^{p^{(m)}}$ is determined by the parameters of the project those between negative pairs to get more powerful protein feature representation, which is more effective to protein fold recognition. To learn the appropriate parameter $W^{M}$ and $B^{M}, W^{M}$ and $B^{M}$ are the ensemble parameters of whole siamese network, we formulate our RFRSN as the following optimization problem:

$$
L\left(Y,\left(Y,\left(x_{1}, x_{2}\right)\right)=\frac{1}{2 N} \sum_{n=1}^{N}\left(Y D_{W}^{2}+(1-Y) \max \left(m-D_{W}^{2}, 0\right)^{2}\right)\right.
$$

Where $D_{W}^{2}$ is the Euclidean distance of the protein $X^{1}$ and $X^{2}$ can be computed as:

$$
D_{W}^{2}=\left\|f\left(x_{i}\right)-f\left(x_{2}\right)\right\|_{2}^{2}=\left(\sum_{i=1}^{p}\left(f\left(X_{1}\right)^{i}-f\left(X_{2}\right)^{i}\right)^{2}\right)^{1 / 2}
$$


Where $p$ is the dimension of the final output by deep neural network. Y is the label whether the two samples come from the same fold type, when two samples share the same fold label, Y is set to 1, and otherwise it will be set to 0. From Equation (3), when $\mathrm{Y}=1$, we just need to get as close as possible between the two samples, when $Y=0$, we need to make the distance between the sample pairs greater than the threshold value margin. Then value of margin has to be assumed before training process. We employ the SGD method to train the entire network.

Assigning fold type to query protein: Due to the high discrimination, the final output feature representation can be used to assess the distance between proteins and can be used to rank template proteins for a target protein. The fold type of the template protein that matches the query protein the most will be assigned to the query protein.

\section{Results}

\section{Evaluation strategy and comparison}

In our experiment, we use top1 and top5 as the measure of our method, Top1 Accuracy refers to the accuracy with which the top-ranked category matches actual labels, Top-5 Accuracy refers to the accuracy with which the top5 categories include actual labels. We use each protein in test set as query protein, compare it with template protein, and final rank them based on the distance.

For SSAfold, we freeze the parameter of network of SSAfold and use it as a feature descriptor, query protein.

For RFRSN method, we also freeze the parameter of network of SSAfold and use it as a feature descriptor, the output of first fully connected layer as protein feature. Then we randomly selected 500,000 pairs of protein samples for training dataset, of which 250,000 were positive samples and 250,000 were negative samples. These pairs of protein samples are used to train the siamese network. Finally, we pass the query protein feature and the template protein feature into the siamese network to computer the distance between two proteins. The fold type of the template protein closest to the query protein is assigned to the query protein. 

SAM-T98 [42], BLASTLINK [19]), SSEARCH [9], SSHMM [43], THREADER [44], Fugue [45],

RF-Fold ), deep learning methods (DN-Fold, DeepFR) and ensemble methods (RFDN-Fold, DN-FoldS, DN-FoldR, TA-fold[53]).

Table 1. Performance comparison of different protein fold recognition methods on the test dataset.

\begin{tabular}{|c|c|c|c|c|c|c|}
\hline \multirow{2}{*}{ Method } & \multicolumn{2}{|c|}{ Family } & \multicolumn{2}{|c|}{ Superfamily } & \multicolumn{2}{|c|}{ Fold } \\
\hline & Top $1(\%)$ & Top $5(\%)$ & Top $1(\%)$ & Top $5(\%)$ & Top $1(\%)$ & Top $5(\%)$ \\
\hline PSI-Blast & 71.2 & 72.3 & 27.4 & 27.9 & 4.0 & 4.7 \\
\hline HMMER [42] & 67.7 & 73.5 & 20.7 & 31.3 & 4.4 & 14.6 \\
\hline SAM-T98 & 70.1 & 75.4 & 28.3 & 38.9 & 3.4 & 18.7 \\
\hline BLASTLINK & 74.6 & 78.9 & 29.3 & 40.6 & 6.9 & 16.5 \\
\hline SSERCH & 68.6 & 75.5 & 20.7 & 32.5 & 5.6 & 15.6 \\
\hline SSHMM & 63.1 & 71.7 & 18.4 & 31.6 & 6.9 & 24.0 \\
\hline THREADER & 49.2 & 58.9 & 10.8 & 24.7 & 14.6 & 37.7 \\
\hline Fugue & 82.2 & 85.8 & 41.9 & 53.2 & 12.5 & 26.8 \\
\hline SPARKS [46] & 81.6 & 88.1 & 52.5 & 69.1 & 28.7 & 47.7 \\
\hline SP3 [48] & 81.6 & 86.8 & 55.3 & 67.7 & 30.8 & 47.4 \\
\hline HHpred [51] & 82.9 & 87.1 & 58.0 & 70.0 & 25.2 & 39.4 \\
\hline SP4 & 80.9 & 86.3 & 57.8 & 57.8 & 30.8 & 53.6 \\
\hline SP5 & 82.4 & 87.6 & 59.8 & 70.0 & 37.9 & 58.7 \\
\hline RAPTOR & 86.6 & 89.3 & 56.3 & 69.0 & 38.2 & 58.7 \\
\hline SPARKS-X & 84.1 & 90.3 & 59.0 & 76.3 & 45.2 & 67.0 \\
\hline BoostThreader & 86.5 & 90.5 & 66.1 & 76.4 & 42.6 & 57.4 \\
\hline FOLDpro & 85.0 & 89.9 & 55.0 & 70.0 & 26.5 & 48.3 \\
\hline RF-Fold & 84.5 & 91.5 & 63.4 & 79.3 & 40.8 & 58.3 \\
\hline DN-Fold & 84.5 & 91.2 & 61.5 & 76.5 & 33.6 & 60.7 \\
\hline DN-FoldS & 84.1 & 91.2 & 62.7 & 76.7 & 33.3 & 57.9 \\
\hline DN-FoldR & 82.3 & 88.3 & 56.0 & 71.0 & 27.4 & 56.7 \\
\hline DeepFR (S1) & 67.4 & 80.9 & 47.0 & 63.4 & 44.5 & 62.9 \\
\hline DeepFR (S2) & 65.4 & 83.4 & 51.4 & 67.1 & 56.1 & 70.1 \\
\hline DeepFRpro (S1) & 85.6 & 91.9 & 66.6 & 82.0 & 57.6 & 73.8 \\
\hline SSAFold & 65.8 & 84.9 & 58.3 & 73.0 & 59.8 & 73.2 \\
\hline RFRSN & 66.3 & 76.1 & 62.4 & 78.6 & 62.0 & 82.6 \\
\hline
\end{tabular}


As in table 1, our proposed SSAFold significantly outperformed all the other methods at the fold level except RFRSN method. Specifically, the accuracy of SSAfold for top 1 and top 5 predictions are $65.8 \%, 84.9 \%, 58.3 \%, 73.0 \%, 59.8 \%$ and $73.2 \%$ at family level, superfamily level and fold level, respectively. Especially compared with DeepFR, the accuracy of SSAFold for top 1 and top 5 at family level is about $7 \%$ and $6 \%$ higher than DeepFR, the fold-feature of DeepFR is the best features for protein fold recognition before, respectively. In addition, since the whole SSAfold model is connected by two neural network models, the entire protein fold recognition model deals directly with the protein sequence, proteins fold type can be identified by SSAfold faster than other methods. For RFRSN method, we learn a new metric distance by siamese network and we employ the new metric distance to measure the query protein and template protein. From the table 1, we can see that the new measure of distance can more effectively measure the relationship between two proteins, and the accuracy of SSAfold for top 1 and top 5 predictions are $66.3 \%, 76.1 \%, 62.4 \%$, $78.6 \%, 62.0 \%, 82.6 \%$ at family level, superfamily level and fold level, respectively. In particular, for some ensemble methods, such as RFDN-Fold, DN-FoldS and DN-FoldR, our proposed SSAfold and RFRSN method still can outperform them, this is attributed to the powerful and automatic feature extraction capability of the convolutional neural network. In addition, potential protein residue-residue relationships contain a lot of structural information also contribute this improvement. For RFRSN method, it learns a right distance metric to make the distance between positive protein pairs is reduced and that of negative pairs be enlarged as much as possible. The ideal of RFRSN is simple and independent, it can be easily used to process other powerful protein feature descriptor for different tasks.

\section{Discussion about margin}

330 For parameter $m$, different parameters have a great influence on the results, and the main factor 331 determining $m$ is the distribution of protein feature representation.

332 Table 1. Performance comparison of different protein fold recognition methods on the test dataset.

\begin{tabular}{ccccccc}
\hline \multirow{2}{*}{$\mathrm{m}$} & \multicolumn{2}{c}{ Family } & \multicolumn{2}{c}{ Superfamily } & \multicolumn{2}{c}{ Fold } \\
\cline { 2 - 7 } & Top 1 (\%) & Top 5 (\%) & Top 1 (\%) & Top 5 (\%) & Top 1 (\%) & Top 5 (\%) \\
\hline 0.2 & 60.4 & 71.2 & 51.2 & 62.2 & 52.6 & 66.0 \\
\hline 0.7 & 67.7 & 86.8 & 60.6 & 75.8 & 62.0 & 75.6 \\
\hline 1.2 & 65.9 & 80.0 & 58.3 & 78.6 & 59.8 & 73.2 \\
\hline \multicolumn{6}{c}{14}
\end{tabular}




\begin{tabular}{lllllll}
\hline 1.7 & 61.0 & 76.8 & 57.2 & 72.6 & 54.2 & 68.0 \\
\hline 2.2 & 58.0 & 72.0 & 51.8 & 68.9 & 50.8 & 62.0 \\
\hline
\end{tabular}

From table 3 , we can see that the setting of margin determines the classification effect. When $m$ is set to 0.7 , we can see that our RFRSN get the best performance, respectively. the accuracy of RFRSN for top 1 and top 5 predictions are 66.3\%, 76.1\%,62.4\%, 78.6\%,62.0\% and 82.6\% at family level, superfamily level and fold level, respectively. However, when the setting of margin does not correspond to the protein feature distribution, a poor effect may be obtained. For example, when $\mathrm{m}$ is set to 0.2 , the performance of RFRSN is not as good as our SSAfold. On the other hand, it is not surprising, when $\mathrm{m}$ is too small, the boundary between positive and negative samples

340 becomes blurred and when $m$ is too big, it is very difficult to learn the parameters of the siamese network.

\section{Feature analysis}

For downstream tasks, deep convolutional neural network is a black box and we don't know why the neural convolutional neural network works even though it does very well on a lot of tasks. In this study, we take protein fold recognition as an example, through the pictorial display of features learned from each convolutional layer, we briefly analyse how these features affect fold recognition as the network depth increases.
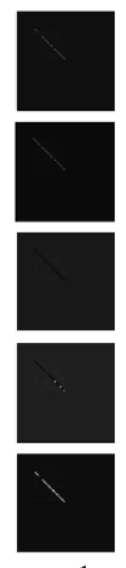

$\operatorname{cov} 1$
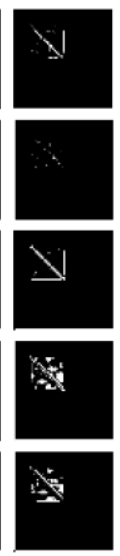

$\operatorname{cov} 2$
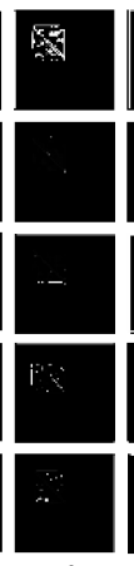

$\operatorname{cov} 3$
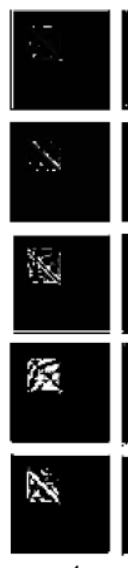

$\operatorname{cov} 4$
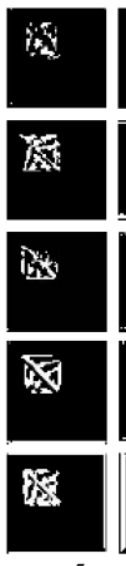

$\operatorname{cov} 5$
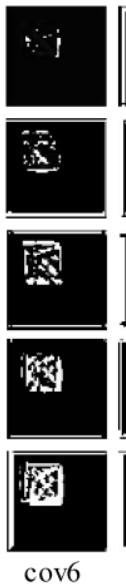
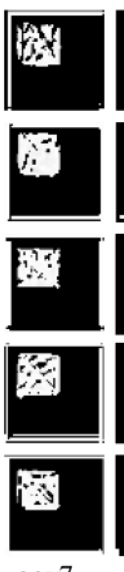

$\operatorname{cov} 7$
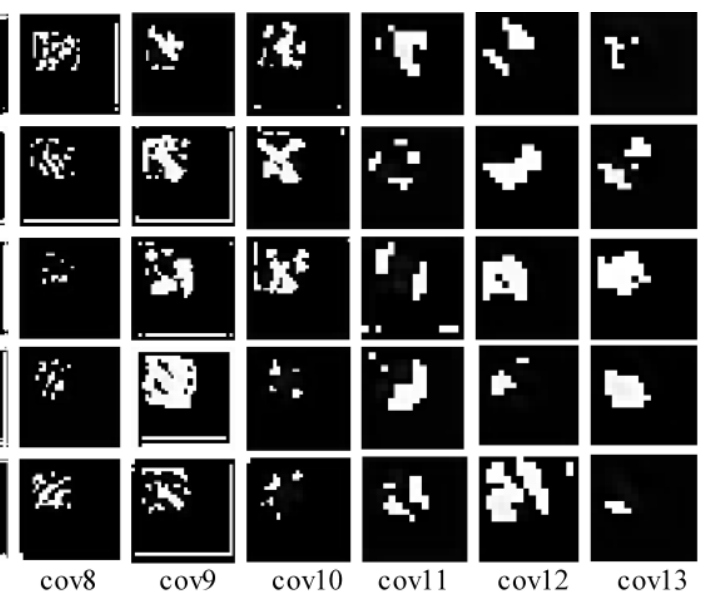

From the Fig. 4, in the early stages of training, the shallow convolutional kernel focuses on the

entire input information (here, it also contains the supplementary 0 element). Now, the features extracted by the shallow convolutional kernel is low-level and contains entire residue points. As the network gets deeper and deeper, the convolutional kernels turn attention into local protein residue that may affect the type of protein fold type, protein residues that have no effect on the 
bioRxiv preprint doi: https://doi.org/10.1101/2021 04.27.441698; this version posted April 29, 2021. The copyright holder for this preprint (which was not certified by peer review) is the author/funder. This article is a US Government work. It is not subject to copyright under 17 USC 105 and is also made available for use under a CCO license.

354 classification results and the complement of 0 are ignored at this stage. In the later stages of training,

355 at this time, the features extracted by convolutional kernel are more abstract and almost difficult to

356 explain. According to our knowledge, these features may be the relationship between two residues

357 in the whole protein chain, the interactions between them affect the protein fold type.

358 


\section{Conclusion}

360 Accurate and fast classification of protein fold is essential for predicting protein tertiary structure.

361 In this paper, we have proposed two complementary methods. SSAfold for extracting robust and 362 discriminative features, it can describe the protein automatically and comprehensively. RFRSN for 363 projecting the feature representation into a fold subspace, where the distance between proteins 364 shared same fold type is closer to the distance between proteins shared different fold type. The 365 protein feature representation processed by RFRSN is very applicable for template-based fold 366 assignment. In addition, the proposed method only using the protein residue- residue relationship 367 and there is no integration of other protein information and other classification algorithms. Even so, 368 our proposed SSAfold and RFRSN has achieved competitive results. 


\section{REFERENCES}

1. Chung I-F, Huang C-D, Shen Y-H et al. Recognition of structure classification of protein folding by NN and SVM hierarchical learning architecture. Artificial Neural Networks and Neural Information Processing-ICANN/ICONIP 2003. Springer, 2003, 1159-1167.

2. Shen H-B, Chou K-C. Ensemble classifier for protein fold pattern recognition, Bioinformatics 2006;22:1717-1722.

3. Liu B, Chen J, Guo $M$ et al. Protein remote homology detection and fold recognition based on Sequence-Order Frequency Matrix, IEEE/ACM Transactions on Computational Biology and Bioinformatics 2017; 16:292-300.

4. Jo T, Cheng J. Improving protein fold recognition by random forest. In: BMC Bioinformatics. 2014, p. S14. Springer.

5. Jo T, Hou J, Eickholt $\mathrm{J}$ et al. Improving protein fold recognition by deep learning networks, Scientific reports 2015;5:17573.

6. Liaw A, Wiener M. Classification and regression by randomForest, R news 2002;2:18-22.

7. Altschul SF, Gish W, Miller W et al. Basic local alignment search tool, Journal of molecular biology 1990;215:403-410.

8. Eddy SR. Profile hidden Markov models, Bioinformatics (Oxford, England) 1998;14:755-763.

9. Pearson WR, Lipman DJ. Improved tools for biological sequence comparison, Proceedings of the National Academy of Sciences 1988;85:2444-2448.

10. Söding J. Protein homology detection by HMM-HMM comparison, Bioinformatics 2005;21:951-960.

11. Peng J, Xu J. Boosting protein threading accuracy. In: Annual International Conference on Research in Computational Molecular Biology. 2009, p. 31-45. Springer.

12. Xu J, Li M, Kim D et al. RAPTOR: optimal protein threading by linear programming, Journal of bioinformatics and computational biology 2003;1:95-117.

13. Zhu J, Zhang H, Li SC et al. Improving protein fold recognition by extracting fold-specific features from predicted residue-residue contacts, Bioinformatics 2017;33:3749-3757.

14. Seemayer S, Gruber M, Söding J. CCMpred-fast and precise prediction of protein residue-residue contacts from correlated mutations, Bioinformatics 2014;30:3128-3130.

15. Bepler T, Berger B. Learning protein sequence embeddings using information from structure, arXiv preprint arXiv:1902.08661 2019.

16. Chandonia J-M, Fox NK, Brenner SE. SCOPe: manual curation and artifact removal in the structural classification of proteins-extended database, Journal of molecular biology 2017;429:348-355.

17. Fox NK, Brenner SE, Chandonia J-M. SCOPe: Structural Classification of Proteins-extended, integrating SCOP and ASTRAL data and classification of new structures, Nucleic acids research 2014;42:D304-D309.

18. Li W, Godzik A. Cd-hit: a fast program for clustering and comparing large sets of protein or nucleotide sequences, Bioinformatics 2006;22:1658-1659.

19. Lindahl E, Elofsson A. Identification of related proteins on family, superfamily and fold level, Journal of molecular biology 2000;295:613-625.

20. Globerson A, Roweis ST. Metric learning by collapsing classes. In: Advances in neural information processing systems. 2006, p. 451-458.

21. Schultz M, Joachims T. Learning a distance metric from relative comparisons. In: Advances in neural 
information processing systems. 2004, p. 41-48.

22. Shalev-Shwartz S, Singer Y, Ng AY. Online and batch learning of pseudo-metrics. In: Proceedings of the twenty-first international conference on Machine learning. 2004, p. 94.

23. Weinberger KQ, Saul LK. Distance metric learning for large margin nearest neighbor classification, Journal of Machine Learning Research 2009;10.

24. Davis JV, Kulis B, Jain P et al. Information-theoretic metric learning. In: Proceedings of the 24th international conference on Machine learning. 2007, p. 209-216.

25. Tsang IW, Kwok JT, Bay C et al. Distance metric learning with kernels. In: Proceedings of the International Conference on Artificial Neural Networks. 2003, p. 126-129. Citeseer.

26. Xiong F, Gou M, Camps O et al. Person re-identification using kernel-based metric learning methods. In: European conference on computer vision. 2014, p. 1-16. Springer.

27. Yeung D-Y, Chang H. A kernel approach for semisupervised metric learning, IEEE Transactions on Neural Networks 2007;18:141-149.

28. $\mathrm{Hu} \mathrm{J}$, Lu J, Tan Y-P. Discriminative deep metric learning for face verification in the wild. In: Proceedings of the IEEE conference on computer vision and pattern recognition. 2014, p. 1875-1882.

29. Hu J, Lu J, Tan Y-P. Deep transfer metric learning. In: Proceedings of the IEEE conference on computer vision and pattern recognition. 2015, p. 325-333.

30. Sun X, Xv H, Dong J et al. Few-shot Learning for Domain-specific Fine-grained Image Classification, IEEE Transactions on Industrial Electronics 2020.

31. Tian $\mathrm{C}, \mathrm{Xu}$ Y, Zuo W. Image denoising using deep CNN with batch renormalization, Neural Networks 2020;121:461-473.

32. Kowsari K, Jafari Meimandi K, Heidarysafa M et al. Text classification algorithms: A survey, Information 2019;10:150.

33. Yao L, Mao C, Luo Y. Graph convolutional networks for text classification. In: Proceedings of the Aaai Conference on Artificial Intelligence. 2019, p. 7370-7377.

34. Mun J, Yang L, Ren $Z$ et al. Streamlined dense video captioning. In: Proceedings of the IEEE Conference on Computer Vision and Pattern Recognition. 2019, p. 6588-6597.

35. Li Y, Hu J, Zhang $\mathrm{C}$ et al. ResPRE: high-accuracy protein contact prediction by coupling precision matrix with deep residual neural networks, Bioinformatics 2019;35:4647-4655.

36. Jones DT, Kandathil SM. High precision in protein contact prediction using fully convolutional neural networks and minimal sequence features, Bioinformatics 2018;34:3308-3315.

37. Derevyanko G, Grudinin S, Bengio Y et al. Deep convolutional networks for quality assessment of protein folds, Bioinformatics 2018;34:4046-4053.

38. Adhikari B. DEEPCON: protein contact prediction using dilated convolutional neural networks with dropout, Bioinformatics 2020;36:470-477.

39. Remmert M, Biegert A, Hauser A et al. HHblits: lightning-fast iterative protein sequence searching by HMM-HMM alignment, Nature methods 2012;9:173-175.

40. LeCun Y, Boser BE, Denker JS et al. Handwritten digit recognition with a back-propagation network. In: Advances in neural information processing systems. 1990, p. 396-404.

41. Chopra S, Hadsell R, LeCun Y. Learning a similarity metric discriminatively, with application to face verification. In: 2005 IEEE Computer Society Conference on Computer Vision and Pattern Recognition (CVPR'05). 2005, p. 539-546. IEEE.

42. Karplus K, Barrett C, Hughey R. Hidden Markov models for detecting remote protein homologies, 
Bioinformatics (Oxford, England) 1998;14:846-856.

43. Hargbo J, Elofsson A. Hidden Markov models that use predicted secondary structures for fold recognition, Proteins: Structure, Function, and Bioinformatics 1999;36:68-76.

44. Jones DT, Taylort W, Thornton JM. A new approach to protein fold recognition, Nature 1992;358:86-89. 45. Shi J, Blundell TL, Mizuguchi K. FUGUE: sequence-structure homology recognition using environment-specific substitution tables and structure-dependent gap penalties, Journal of molecular biology 2001;310:243-257.

46. Zhou H, Zhou Y. Single-body residue-level knowledge-based energy score combined with sequenceprofile and secondary structure information for fold recognition, Proteins: Structure, Function, and Bioinformatics 2004;55:1005-1013.

47. Yang JY, Chen X. Improving taxonomy-based protein fold recognition by using global and local features, Proteins: Structure, Function, and Bioinformatics 2011;79:2053-2064.

48. Zhou H, Zhou Y. Fold recognition by combining sequence profiles derived from evolution and from depth-dependent structural alignment of fragments, Proteins: Structure, Function, and Bioinformatics 2005;58:321-328.

49. Liu S, Zhang C, Liang S et al. Fold recognition by concurrent use of solvent accessibility and residue depth, Proteins: Structure, Function, and Bioinformatics 2007;68:636-645.

50. Zhang W, Liu S, Zhou Y. Sp 5: improving protein fold recognition by using torsion angle profiles and profile-based gap penalty model, PloS one 2008;3:e2325.

51. Söding J, Biegert A, Lupas AN. The HHpred interactive server for protein homology detection and structure prediction, Nucleic acids research 2005;33:W244-W248.

52. Xu D, Jaroszewski L, Li Z et al. FFAS-3D: improving fold recognition by including optimized structural features and template re-ranking, Bioinformatics 2014;30:660-667.

53. Xia J, Peng Z, Qi D et al. An ensemble approach to protein fold classification by integration of template-based assignment and support vector machine classifier, Bioinformatics 2017;33:863-870.

54. Cheng J, Baldi P. A machine learning information retrieval approach to protein fold recognition, Bioinformatics 2006;22:1456-1463. 
bioRxiv preprint doi: https://doi.org/10.1101/2021.04.27.441698; this version posted April 29, 2021. The copyright holder for this preprint (which was not certified by peer review) is the author/funder. This article is a US Government work. It is not subject to copyright under 17 USC 105 and is also made available for use under a CCO license. 